\title{
Disrupted Association Between Empathy and Brain Structure in Attention-Deficit/Hyperactivity Disorder
}

\author{
Jeonghwan Lee, ${ }^{1,2}$ Jung-Woo Son ${ }^{1,2}$, Siekyeong $\mathrm{Kim}^{1,2}$, Ji-eun $\mathrm{Kim}^{3}$, Seungwon Chung, \\ Hei-Rhee Ghim ${ }^{4}$, Sang-Ick Lee ${ }^{1,2}$, Chul-Jin Shin ${ }^{1,2}$, and Gawon Ju', \\ 1 Department of Psychiatry, Chungbuk National University Hospital, Cheongju, Korea \\ ${ }^{2}$ Department of Neuropsychiatry, College of Medicine, Chungbuk National University, Cheongju, Korea \\ ${ }^{3}$ Ma-Eum Pyong-On Psychiatric Clinic, Yongin, Korea \\ ${ }^{4}$ Department of Psychology, Chungbuk National University, Cheongju, Korea
}

Objectives: To investigate the relationship between brain structure and empathy in early adolescents with attention-deficit/hyperactivity disorder (ADHD).

Methods: Nineteen early adolescents with ADHD and 20 healthy controls underwent 3T MRI. All the participants were assessed for different aspects of empathy using measures including the Interpersonal Reactivity Index and Empathy Quotient. Cortical thickness and subcortical structural volume based on T1-weighted scans were analyzed using FreeSurfer.

Results: Cognitive empathy ( $\mathrm{t}=-2.52, \mathrm{p}=0.016)$ and perspective taking $(\mathrm{t}=-2.10, \mathrm{p}=0.043)$ were impaired in the ADHD group compared with the control group. The cluster encompassing the left posterior insular, supramarginal, and transverse temporal cortices [cluster-wise p-value $(\mathrm{CWP})=0.001$, which are associated with emotional empathy, was significantly smaller in the ADHD group, and the volume of the left nucleus accumbens was greater than that of the control group $(\mathrm{F}=10.12, \mathrm{p}=0.003$, effect size $=0.22)$. In the control group, the left superior temporal $(\mathrm{CWP}=0.002)$ and lingual cortical $(\mathrm{CWP}=0.035)$ thicknesses were positively associated with cognitive empathy, while the right amygdala volume was positively associated with empathic concern (Coef=14.26, $t=3.92, p=0.001$ ). However, there was no significant correlation between empathy and brain structure in the ADHD group.

Conclusion: The ADHD group had a smaller volume of the cortical area associated with emotional empathy than the control group, and there was no brain region showing significant correlation with empathy, unlike in the control group.

Keywords: Attention-deficit/hyperactivity disorder; Adolescent; Neuroimaging; Brain cortical thickness; Empathy.

Received: March 26, 2021 / Revision: April 25, 2021 / Accepted: May 7, 2021

Address for correspondence: Jung-Woo Son, Department of Neuropsychiatry, College of Medicine, Chungbuk National University, 1 Chungdae-ro, Seowon-gu, Cheongju 28644, Korea

Tel: +82-43-269-6187, Fax: +82-43-267-7951, E-mail: mammosss@hanmail.net

\section{INTRODUCTION}

Attention-deficit/hyperactivity disorder (ADHD) is a relatively common neuropsychiatric disorder in children and adolescents characterized by symptoms such as inattention, hyperactivity, and impulsivity. Children with ADHD experience peer rejection more frequently [1], have problems interacting with peers, and demonstrate poorer social function [2] in school than typically developing children. Although the poor social function in ADHD has been attributed to inattention [1], misrecognizing the emotions of other people also plays a critical role. The recognition of all emotions, except anger, through facial expressions, voices, and stories by children with ADHD is less accurate; however, this misidentification of emotions is

This is an Open Access article distributed under the terms of the Creative Commons Attribution Non-Commercial License (https://creativecommons.org/licenses/by-nc/4.0) which permits unrestricted non-commercial use, distribution, and reproduction in any medium, provided the original work is properly cited. random, rather than a constant tendency, and linked to impaired empathic response [3].

Empathy is an important motivator and mediator of human social behavior. According to teacher and parent reporting scales, children with ADHD are less empathic [4]. Empathy has cognitive and emotional components. To cognitively empathize, an individual should have a mental representation of the perspectives of others, known as the theory of mind (ToM) [5]. Emotional empathy requires emotional contagion, which refers to identifying with the emotional state of others, which is recognized through observation or imagination [6].

Research on adults has shown that the ventromedial prefrontal cortex, temporoparietal junction, and superior temporal sulcus are associated with cognitive empathic response, whereas the inferior frontal gyrus, insula, and inferior parietal lobule are associated with emotional empathy [7]. A study involving children reported that brain regions recruited during 
empathic responses change with age; for example, the activities of the posterior insula and amygdala are higher and the engagements of the inferior and superior frontal gyri are lower in younger individuals [8]. To the best of our knowledge, few studies have investigated the association between brain structure and prosocial behavior in children with ADHD [9]; however, none of them investigated the association between empathy and brain structure in young adolescents with ADHD. Since ADHD is a neurodevelopmental disorder associated with structural and functional brain alterations, the relationship between empathy and brain structure in children with ADHD may differ from that in typically developing children. Based on this, we aimed to investigate the empathic response of young adolescents with ADHD and its correlation with brain structure.

\section{METHODS}

\section{Participants and measurements}

Young adolescents with ADHD were recruited from child and adolescent psychiatry clinics in Cheongju and Seoul, South Korea. Typically developing healthy volunteers were recruited through advertising in middle schools and communities. The common inclusion criteria for the two groups were as follows: 1) age of 12-15 years, 2) intelligence index of $\geq 80$ according to the short form of the Korean Weschler Intelligence Scale for children [10], 3) absence of physical illnesses, and 4) righthandedness. In addition, the Kiddie-Schedule for Affective Disorders and Schizophrenia-Present and Lifetime VersionKorean Version (K-SADS-PL-K) [11] was used to assess all the participants. All the participants in the ADHD group met the ADHD diagnosis criteria; they did not show any other major mental illness, such as schizophrenia, bipolar disorder, major depression, and substance use disorder, and they were taking stimulant medications before participation in the study. The participants in the control group were limited to the absence of disease, as defined by the K-SADS-PL-K diagnostic criteria. Participants were excluded from the control group if they had a history of inattention or hyperactivity despite not satisfying the full criteria for ADHD diagnosis. The final analysis involved 19 participants with ADHD and 20 controls. This study was approved by the Bioethics Committee of Chungbuk National University Hospital (2012-09-083), and the participants signed a written consent form after receiving information on the purpose and method of the study.

The Korean ADHD Rating Scale-IV (K-ARS) was used to quantify the core ADHD symptoms of all the participants. The K-ARS, a tool for evaluating ADHD symptoms, consists of 18 questions covering the ADHD diagnostic criteria of the DSM-IV. The K-ARS uses a Likert scale from 0 to 3 to score the ADHD symptoms over the previous six months [12], and it has subscales such as those for inattentiveness (K-ARS-I) and hyperactivity-impulsivity (K-ARS-H). The components of the empathic ability of the study participants were assessed using the Interpersonal Reactivity Index (IRI). The IRI evaluates cognitive and emotional empathic processes using a 5 -point Likert scale, and it consists of 28 questions [13]. This study used three subscales: perspective taking, which evaluates the tendency to adopt the perspective of others; fantasy, the tendency to transpose oneself into the feelings and actions of fictitious characters; and empathic concern, the tendency to experience feelings of warmth, compassion, and concern for other people. Although the IRI was developed for adults, this study used a translated version of the IRI adapted for use with children, as was done in a previous study [14]. In addition to using the IRI, the empathy of each participant was also assessed using the children's version of the Empathy Quotient (EQ-C) report [15]. In this study, questions for emotional empathy (EQ-C-EE) and cognitive empathy (EQ-C-CE) were translated to Korean, and they were transformed to allow for self-completion.

\section{MRI acquisition and preprocessing}

MRI data were acquired with a Siemens 3T MR scanner (MAGNETOM TrioTim syngo MR B17, Siemens AG, Erlangen, Germany) at the Brain Imaging Center of Korea University. The acquisition parameters were as follows: repetition time $=1900 \mathrm{~ms}$, echo time $=2.52 \mathrm{~ms}$, flip angle $=9^{\circ}$, field of view $=$ $256 \times 256 \mathrm{~mm}$, slice gap $=1 \mathrm{~mm}$. Based on the sagittal T1-weighted images, subcortical segmentation and cortical parcellation were processed using the FreeSurfer software package (version 6.0; https://surfer.nmr.mgh.harvard.edu). The details of the general process are described elsewhere [16]. After registering the T1-weighted magnetization prepared rapid gradient echo image in the standardized Montreal Neurological Institute (MNI) atlas, the white and gray matter were distinguished based on the voxel locations on the MNI atlas and the signal strength in and around it. Next, the non-brain tissue was removed using a hybrid watershed algorithm. Thereafter, automated Talairach transformation was performed, and the subcortical white and deep gray matter were segmented. All the reconstructed brain images were visually inspected and corrected for errors using intensity normalization or the addition of control points.

\section{Statistical analysis}

The CRAN R statistical package version 3.6.1. (https://www. r-project.org) was used for statistical analysis. The outcomes of the assessments with the intelligence scale, K-ARS, EQ-C, and IRI measures were compared using Student's t-test or the 
Mann-Whitney test depending on normality and uniformity, and the sex ratio was compared using the chi-squared test. For the analysis of cortical thickness and volume, the cortical surface map of full width at half-maximum Gaussian kernel smoothed at $15 \mathrm{~mm}$ was used. We compared the thicknesses and volumes of the cerebral cortices of the two groups and used a linear regression model to determine the relationship with the scores of K-ARS, EQ-C, and IRI. Sex and age were set as confounding variables for the analysis of brain structure. To reduce the possibility of false-positives, the voxel-wise threshold was set to $\mathrm{p}<0.001$, and the cluster-wise correction for multiple comparisons was applied with $\mathrm{p}<0.05$.

The volumes of the subcortical structures were normalized before computing between-groups comparison and correlations with the scales. The normalized volume was derived using the following formula:

Volume $_{\text {adj }}=$ Volume $_{\text {nat }}-\mathrm{b}\left(\mathrm{eTIV}_{\text {nat }}-\right.$ mean eTIV $\left.\mathrm{V}_{\text {nat }}\right)$ where Volume adj is the corrected volume, Volume $e_{\text {nat }}$ is the volume in the native space, and b is the slope of the volume regression on the estimated intracranial volume (eTIV). The mean eTIV is the mean eTIV for all samples. All volumes reported in the Results section correspond to the Volume ${ }_{\text {adj. }}$.

The subcortical structural volumes are known to be affected by age and sex. Therefore, analysis of covariance (ANCOVA) was used to compare the volumes for the groups using age and sex as covariates. A significance level of $\mathrm{p}<0.007$ (Bonferroni multiple comparisons correction $\mathrm{p}<0.05 / 7$ ) was used for the comparison of seven subcortical volumes in each hemisphere. Since the amygdala is known to be functionally and structurally associated with empathy, the association between its volume and the measures of empathy for each group was determined using multiple linear regression analysis, with age and sex as covariates.

\section{RESULTS}

\section{Demographic characteristics and scales}

The average age was $13.47 \pm 0.90$ years in the ADHD group and $13.35 \pm 1.18$ years in the control group, and the IQ was $113.84 \pm 11.47$ in the ADHD group and $110.70 \pm 15.17$ in the control group; the differences were not significant (Table 1). Additionally, the sex ratio did not significantly differ in the groups [ADHD group (M:F)=14:5, Control group=11:9]. The total K-ARS ( $\mathrm{u}=4.58, \mathrm{p}=4.67 \mathrm{E}-06)$, K-ARS-I $(\mathrm{u}=4.70, \mathrm{p}=$ 2.58E-06), and K-ARS-H ( $\mathrm{u}=3.98, \mathrm{p}=6.82 \mathrm{E}-05)$ scores were significantly higher in the ADHD group than in the control group. Although the total IRI score and the fantasy and empathic concern subscales were not significantly different in the two groups, the perspective taking subscale was significantly lower in the ADHD group $(\mathrm{t}=-2.10, \mathrm{p}=0.043)$. Additionally, the EQ-C total $(\mathrm{t}=-2.26, \mathrm{p}=0.030)$ and $\mathrm{EQ}-\mathrm{C}-\mathrm{CE}(\mathrm{t}=-2.52$, $\mathrm{p}=0.016$ ) scores were significantly lower in the $\mathrm{ADHD}$ group, whereas there was no significant difference in the EQ-C-EE.

\section{Cortical thickness and volume}

The comparison of the cortical thicknesses and volumes of the two groups, with age and sex as covariates, showed that the volume of the cluster encompassing the posterior insular, supramarginal, and transverse temporal cortices (Brodmann Area 40, 41, 42) was significantly smaller in the ADHD group than in the control group [cluster-wise $\mathrm{p}$-value $(\mathrm{CWP})=0.001$ ] (Fig. 1). The thicknesses of the superior temporal (Brodmann Area 22) $(\mathrm{CWP}=0.002)$ and lingual (Brodmann Area 19) $(\mathrm{CWP}=0.035)$ cortices in the left hemisphere were positively correlated with the EQ-C-CE scores in the control group (Fig. 2). However, no cortical region showed a significant relationship with the empathy scores in the ADHD group.

Table 1. Comparisons of demographic variables and scales

\begin{tabular}{lcccc}
\hline \multicolumn{1}{c}{ Variable } & ADHD $(\mathrm{n}=19)$ & Control $(\mathrm{n}=20)$ & t or U & $\mathrm{p}$ \\
\hline Sex, male & 14 & 11 & $0.38^{*}$ & 0.378 \\
Age $(\mathrm{yr})$ & $13.47 \pm 0.90$ & $13.35 \pm 1.18$ & $0.50^{\dagger}$ & 0.618 \\
IQ & $113.84 \pm 11.47$ & $110.70 \pm 15.17$ & 0.73 & 0.471 \\
ADHD Rating Scale & $25.21 \pm 10.27$ & $6.25 \pm 7.97$ & $4.58^{\dagger}$ & $4.67 \mathrm{E}-06$ \\
$\quad$ Inattentiveness & $15.42 \pm 4.72$ & $3.95 \pm 4.67$ & $4.70^{\dagger}$ & $2.58 \mathrm{E}-06$ \\
$\quad$ Hyperactivity & $9.79 \pm 6.08$ & $2.30 \pm 3.50$ & $3.98^{\dagger}$ & $6.82 \mathrm{E}-05$ \\
Empathy Quotient & $21.32 \pm 7.06$ & $26.95 \pm 8.44$ & -2.26 & 0.030 \\
$\quad$ Cognitive empathy & $8.37 \pm 3.74$ & $11.45 \pm 3.90$ & -2.52 & 0.016 \\
$\quad$ Emotional empathy & $7.95 \pm 4.24$ & $9.10 \pm 3.80$ & -0.90 & 0.376 \\
Interpersonal Reactivity Index & $47.42 \pm 14.99$ & $55.25 \pm 11.55$ & -1.83 & 0.075 \\
$\quad$ Fantasy & $17.21 \pm 6.31$ & $18.90 \pm 4.30$ & -0.98 & 0.333 \\
Perspective taking & $13.68 \pm 5.23$ & $17.05 \pm 4.77$ & -2.10 & -1.59 \\
Empathic concern & $16.53 \pm 6.27$ & $19.30 \pm 4.54$ & 0.043 & 0.121
\end{tabular}

Data are presented as mean \pm standard deviation or $n$. t-value from Student t-test or U-value from Mann-Whitney test. ${ }^{*}$ chisquare test, ${ }^{\dagger}$ Mann-Whitney test. ADHD, attention-deficit/hyperactivity disorder 


\section{Subcortical volume}

ANCOVA showed that the volume of the left nucleus accumbens in the ADHD group was significantly greater than that in the control group $(\mathrm{F}=10.12, \mathrm{p}=0.003$, effect size $=0.22)$ (Table 2). The empathic concern of the IRI showed a positive

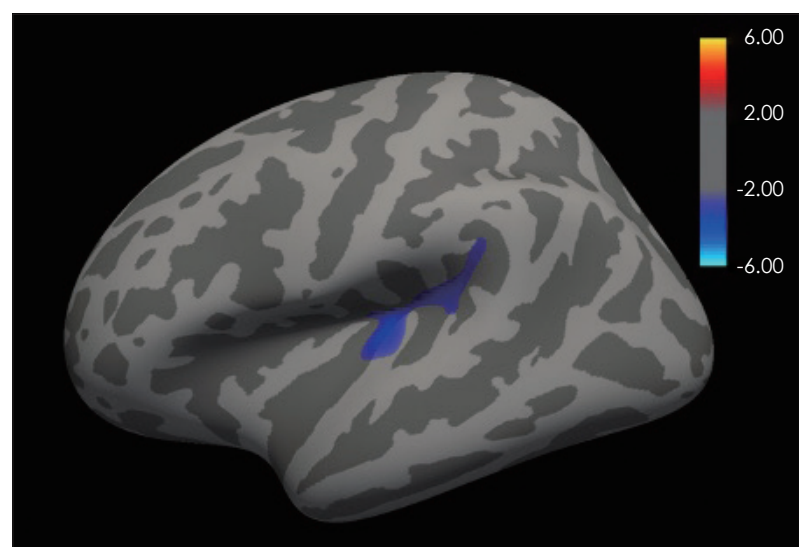

Fig. 1. Group comparisons of cortical volume. Montreal Neurological Institute coordinate: $x=-34.8, y=-28.6, z=7.5$; cluster-wise $\mathrm{p}$-value $=0.001$; annotation=posterior insula, supramarginal, and transverse temporal cortex (Brodmann Area 40, 41, 42) of the left hemisphere. association with the right amygdala volume in the control group (Coef=14.26, $\mathrm{t}=3.92, \mathrm{p}=0.001$ ) (Fig. 3). In the ADHD group, a larger right hippocampal volume was associated with a lower K-ARS-I score (Coef $=-44.55, \mathrm{t}=2.49, \mathrm{p}=0.025)$ (Fig. 4).

\section{DISCUSSION}

In the $\mathrm{ADHD}$ group, emotional empathy, evaluated using the EQ-C-EE, and the empathic concern subscale was not impaired. However, the EQ-C-CE and perspective taking subscale scores, both of which evaluate cognitive empathy, were significantly lower in the ADHD group than in the control group. This finding differs from that of adults with ADHD showed impaired emotional empathy [17]. Perspective taking is essential in assessing cognitive empathic responses. Perspective taking is the ability to adopt the perspective of another person, which is a top-down process necessary for understanding the feelings of others. Our data indicate that the ADHD group had a lower ability of adopting the perspectives of others and cognitively empathizing than the control group. Previous studies involving children with ADHD demonstrated that difficulties in maintaining peer relationships were relat-
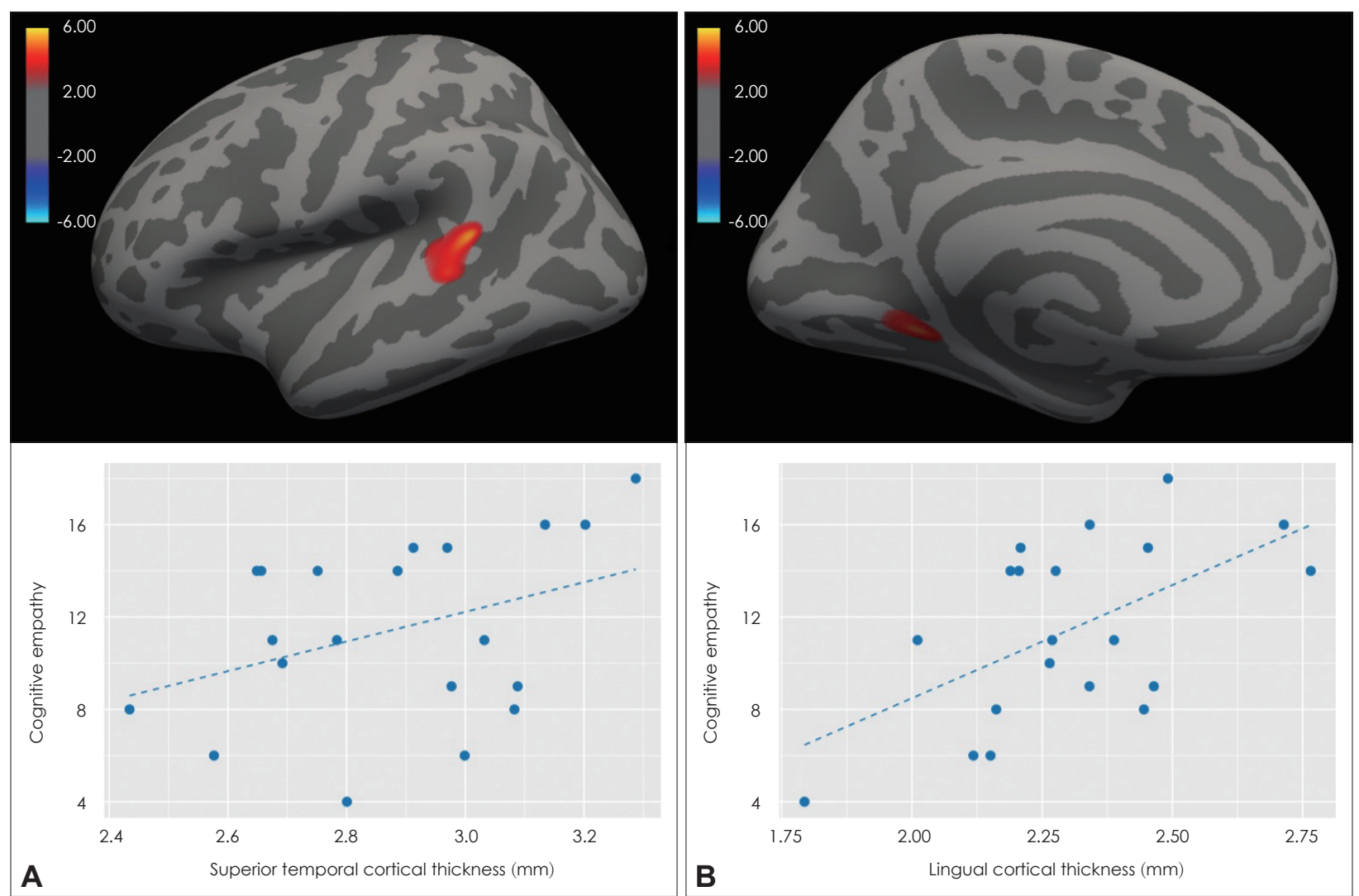

Fig. 2. Association between cortical thickness and cognitive empathy in the control group (Empathy Quotient). A: Cluster 1: MNI coordinate: $x=-62.8, y=-43.8, z=12.0 ; C W P=0.002$; annotation=left superior temporal cortex (Brodmann Area 22) of the left hemisphere (lateral view). B: Cluster 2: MNI coordinate: $x=-18.8, y=-50.7, z=-8.2 ; C W P=0.035$; annotation=left lingual cortex (Brodmann Area 19) in the left hemisphere (medial view). MNI, Montreal Neurological Institute; CWP, cluster-wise p-value. 
Table 2. Comparisons of subcortical structural volumes

\begin{tabular}{|c|c|c|c|c|c|}
\hline & \multirow{2}{*}{$\operatorname{ADHD}\left(\mathrm{mm}^{3}\right)$} & \multirow{2}{*}{ Control $\left(\mathrm{mm}^{3}\right)$} & \multicolumn{3}{|c|}{ ANCOVA } \\
\hline & & & $\mathrm{F}$ & $\mathrm{p}$ & es \\
\hline \multicolumn{6}{|l|}{ Left } \\
\hline Thalamus & $8499.76 \pm 633.31$ & $8374.59 \pm 648.84$ & 0.55 & 0.462 & 0.01 \\
\hline Caudate & $3789.35 \pm 569.37$ & $3661.30 \pm 317.26$ & 1.16 & 0.289 & 0.02 \\
\hline Putamen & $5428.86 \pm 591.91$ & $5039.27 \pm 387.45$ & 5.98 & 0.020 & 0.15 \\
\hline Pallidum & $2228.89 \pm 168.11$ & $2182.66 \pm 129.88$ & 1.12 & 0.297 & 0.03 \\
\hline Hippocampus & $4228.46 \pm 312.67$ & $4137.33 \pm 236.86$ & 0.93 & 0.342 & 0.03 \\
\hline Amygdala & $1837.56 \pm 145.47$ & $1751.96 \pm 116.85$ & 5.04 & 0.031 & 0.11 \\
\hline Nucleus accumbens & $497.02 \pm 70.15$ & $426.22 \pm 72.22$ & 10.12 & $0.003^{*}$ & 0.22 \\
\hline \multicolumn{6}{|l|}{ Right } \\
\hline Thalamus & $7969.97 \pm 473.80$ & $7792.65 \pm 361.76$ & 1.61 & 0.213 & 0.05 \\
\hline Caudate & $3871.71 \pm 487.87$ & $3635.26 \pm 298.87$ & 4.44 & 0.042 & 0.09 \\
\hline Putamen & $5495.81 \pm 574.29$ & $5230.89 \pm 341.03$ & 3.33 & 0.077 & 0.09 \\
\hline Pallidum & $2104.54 \pm 176.93$ & $2050.30 \pm 142.51$ & 1.37 & 0.250 & 0.03 \\
\hline Hippocampus & $4443.99 \pm 382.28$ & $4346.82 \pm 177.41$ & 1.20 & 0.281 & 0.03 \\
\hline Amygdala & $1915.23 \pm 169.98$ & $1841.27 \pm 89.97$ & 4.54 & 0.040 & 0.09 \\
\hline Nucleus accumbens & $568.08 \pm 86.72$ & $530.85 \pm 86.25$ & 1.50 & 0.228 & 0.05 \\
\hline
\end{tabular}

Data are presented as mean \pm standard deviation. * regions remaining significant after controlling for multiple comparisons with Bonferroni correction. ANCOVA, analysis of covariance with age, sex as covariates; es, effect size; ADHD, attention-deficit/hyperactivity disorder

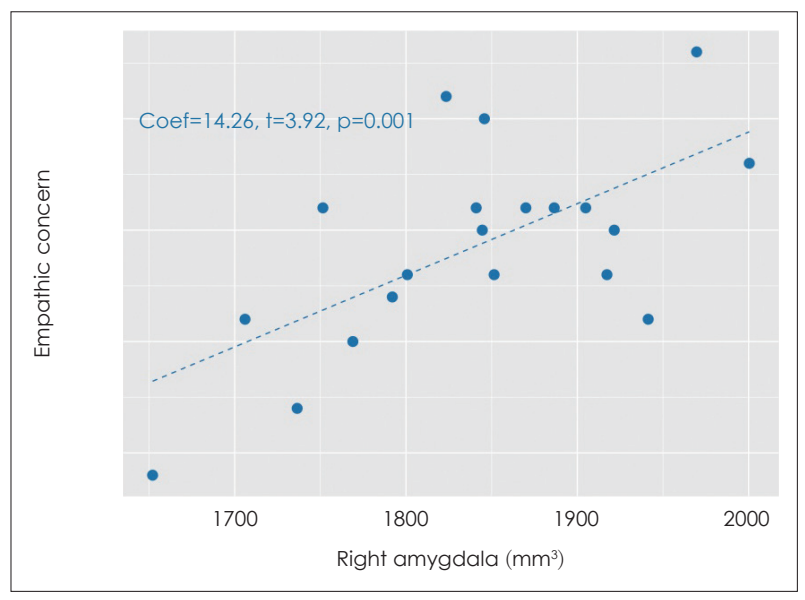

Fig. 3. Association between amygdala volume and empathy in the control group. Multiple linear regression analysis was conducted with age and sex set as covariates.

ed to problems with perspective taking [18], which is disturbed by inattentiveness [19].

After controlling for the effects of age and sex, the ADHD group showed a significantly smaller volume of the cluster encompassing the posterior insular, supramarginal, and the transverse temporal cortices in the left hemisphere, which are associated with emotional empathic responses [7], whereas the emotional empathy scores of the control group showed a positive correlation with the volume of the amygdala, which is known to be involved in empathic responses [20]. In voxelbased morphometry study in patients with alexithymia with

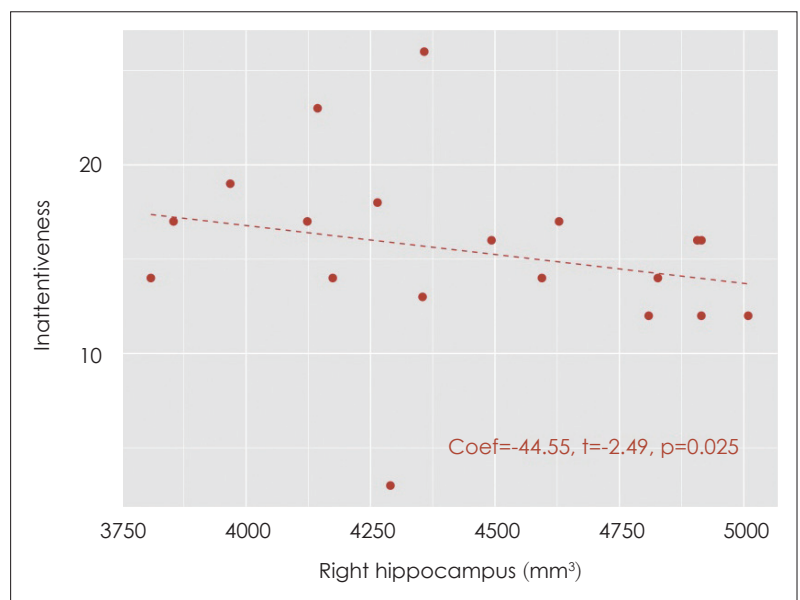

Fig. 4. Association between hippocampal volume and inattentiveness in the attention-deficit/hyperactivity disorder group. Multiple linear regression analysis was conducted with age and sex set as covariates.

deficits in recognizing emotions and impaired empathy toward others, the amygdala volume was reportedly lesser than that of the control group and positively correlated with the empathy level, which was consistent with the results of our study [21]. Various studies have reported that the anterior insula is the brain region commonly activated during emotional empathic response [20]. However, in late adolescents, functional activation of the anterior insula is associated with lower empathic accuracy [22]. In addition, a study involving children and adults showed a stronger activation in the anterior insula with increasing age during empathic responses, suggesting a 
different recruitment pattern of the insular cortex during empathic responses with age [8]. According to Li et al. [23], the insula is activated during emotional empathic responses, but its volume is inversely correlated with empathic responses in young adults. In our study, the ADHD group had a smaller volume of the cortical area associated with the emotional empathic response than the control group, but emotional empathy was not significantly impaired. The possible explanation for this is that the large insula or its functional recruitment in early adolescents does not ensure appropriate emotional empathic responses. In future studies, it will be interesting to observe how the functional activation of emotional empathyrelated areas is associated with the intensity and accuracy of empathic responses in individuals with ADHD.

In the control group alone, a higher cognitive empathy was associated with a thicker superotemporal and lingual cortex in the left hemisphere. The temporoparietal junction is the core ToM region, and it shows a strong functional activity when adopting the perspective of another person suffering from pain or discriminating self from others [24]. Kral et al. [22] found that the functional activation of the temporoparietal junction in late adolescents was related to cognitive empathy, whereas increased functional activity in the adjacent superotemporal sulcus was related to higher empathic accuracy. A study involving young adults also reported that cognitive empathy is related to the functional activation of the superotemporal cortex [25], and young adults with a high empathic response showed lingual cortical thickening [26]. Consistent with the findings in adults, a relationship between brain structure and cognitive empathy was found in the control group in this study.

In the ADHD group, no brain region had a significant relationship with empathy, possibly because ADHD is a neurodevelopmental disorder affecting brain structure. Existing findings on structural brain alterations associated with ADHD vary with the age of the study participants. Based on the analysis of the region of interest of a large sample, the patients with ADHD aged $<14$ years showed cortical thinning of the premotor areas or part of the temporal lobe, but these differences could not be found in late adolescents and adults with ADHD [27]. According to a meta-analysis of participants of all age ranges, the subcortical volume difference between the participants with ADHD and the healthy controls decreases with age [28]. This may be due to the effects of delayed brain maturation; therefore, the brain structural characteristics of $\mathrm{ADHD}$ may differ with age, disease duration, and treatment response.

The participants in the ADHD group had significantly greater left nucleus accumbens volumes than those in the control group. Stimulant treatment increases dopamine release in the striatum, which is associated with an improvement in attention [29]. Structurally, larger volumes of the nucleus accum- bens were associated with better prognoses for using stimulant medications. Furthermore, the volume of the nucleus accumbens increases with longer durations of stimulant treatment in children and young adolescents with ADHD [30]. The increased nucleus accumbens volume presently found in the ADHD group reflects this.

The hippocampal volume in the ADHD group was not different from that in the control group, but the right hippocampal volume was positively correlated with inattentiveness. Chronic low doses of stimulant medications increase hippocampal neurogenesis and cell survival [31]. In a study with more than half of ADHD patients undergoing stimulant treatment, their hippocampal volumes were greater than that of the control group and associated with fewer symptoms [32]. The negative correlation between hippocampal volume and inattentiveness identified in this study may be attributed to the effects of the stimulant medication.

Our study had several limitations. The study participants were relatively few. Accordingly, it was not possible to conduct separate analyses for sex, subtype, or comorbidities such as depression or behavioral problems. Most brain imaging studies have a common limitation; a large sample of participants is difficult to obtain, and taking simultaneous brain images on a large scale is difficult. There was the possibility of falsepositives, and our study reduced the possibility by setting the vertex level threshold for the surface-based analysis to $\mathrm{p}<0.001$ and applying Bonferroni correction to the subcortical volumetric comparisons. In addition, by controlling for age and sex, factors other than ADHD that could affect brain structure were minimized. Therefore, the results of this study should be limited to individuals between the ages of 12 and 15 years, and they cannot be generalized. Second, disease duration or treatment conditions were not controlled for the $\mathrm{ADHD}$ group analysis. As described earlier, ADHD is a neurodevelopmental disorder that affects brain maturation; therefore, individual brain structural characteristics may vary with the disease condition.

\section{CONCLUSION}

Our study on the relationship between structural brain changes and empathy in early adolescents with ADHD showed that the ADHD group had smaller left posterior insular and supramarginal cortices, a larger left nucleus accumbens volume, and no brain region showing a significant correlation with empathy. In contrast, cognitive empathy was positively correlated with the left superotemporal cortical thickness and emotional empathy was correlated with the volume of the right in the control group. In future studies, it will be interesting to observe how the functional activation of emotional empathy- 
related areas is associated with the magnitude and accuracy of empathic responses in individuals with $\mathrm{ADHD}$.

\section{Availability of Data and Material}

The datasets generated or analyzed during the study are available from the corresponding author on reasonable request.

\section{Conflicts of Interest}

Jung-Woo Son, a contributing editor of the Journal of the Korean Academy of Child and Adolescent Psychiatry, was not involved in the editorial evaluation or decision to publish this article. All remaining authors have declared no conflicts of interest.

\section{Author Contributions}

Conceptualization: Jeonghwan Lee, Jung-Woo Son, Siekyeong Kim, Jieun Kim, Hei-Rhee Ghim. Data curation: Jeonghwan Lee, Jung-Woo Son, Ji-eun Kim. Formal analysis: Jeonghwan Lee, Jung-Woo Son. Funding acquisition: Jung-Woo Son, Hei-Rhee Ghim. Investigation: Ji-eun Kim. Methodology: Jeonghwan Lee, Jung-Woo Son, Ji-eun Kim, Seungwon Chung, Hei-Rhee Ghim, Sang-Ick Lee, Chul-Jin Shin, Gawon Ju. Project administration: Jung-Woo Son, Siekyeong Kim, Hei-Rhee Ghim, Sang-Ick Lee, Chul-Jin Shin, Gawon Ju. Resources: Jung-Woo Son, Siekyeong Kim, Ji-eun Kim, Hei-Rhee Ghim, Sang-Ick Lee, Chul-Jin Shin, Gawon Ju. Software: Jeonghwan Lee, Siekyeong Kim. Supervision: Siekyeong Kim, HeiRhee Ghim, Sang-Ick Lee, Chul-Jin Shin, Gawon Ju. Validation: Jung-Woo Son, Siekyeong Kim, Seungwon Chung. Visualization: Jeonghwan Lee. Writing-original draft: Jeonghwan Lee. Writing-review \& editing: Jeonghwan Lee, Jung-Woo Son, Seungwon Chung.

\section{ORCID iDs}

$\begin{array}{ll}\text { Jeonghwan Lee } & \text { https://orcid.org/0000-0002-9533-848X } \\ \text { Jung-Woo Son } & \text { https://orcid.org/0000-0003-4972-3923 } \\ \text { Siekyeong Kim } & \text { https://orcid.org/0000-0001-8466-9649 } \\ \text { Ji-eun Kim } & \text { https://orcid.org/0000-0001-6837-0137 } \\ \text { Seungwon Chung } & \text { https://orcid.org/0000-0002-3009-2722 } \\ \text { Hei-Rhee Ghim } & \text { https://orcid.org/0000-0002-9455-3268 } \\ \text { Sang-Ick Lee } & \text { https://orcid.org/0000-0001-5134-8064 } \\ \text { Chul-Jin Shin } & \text { https://orcid.org/0000-0001-7618-7201 } \\ \text { Gawon Ju } & \text { https://orcid.org/0000-0002-5358-4578 }\end{array}$

\section{Funding Statement \\ None}

\section{Acknowledgments}

The preliminary findings of this study were presented as an Eposter at the 33rd European College of Neuropsychopharmacology Congress.

\section{REFERENCES}

1) Stenseng F, Belsky J, Skalicka V, Wichstrøm L. Peer rejection and attention deficit hyperactivity disorder symptoms: reciprocal relations through ages 4, 6, and 8. Child Dev 2016;87:365-373.

2) Nijmeijer JS, Minderaa RB, Buitelaar JK, Mulligan A, Hartman CA, Hoekstra PJ. Attention-deficit/hyperactivity disorder and social dysfunctioning. Clin Psychol Rev 2008;28:692-708.

3) Cadesky EB, Mota VL, Schachar RJ. Beyond words: how do children with ADHD and/or conduct problems process nonverbal information about affect? J Am Acad Child Adolesc Psychiatry 2000; 39:1160-1167.

4) Deschamps PK, Schutter DJ, Kenemans JL, Matthys W. Empathy and prosocial behavior in response to sadness and distress in 6- to 7-year olds diagnosed with disruptive behavior disorder and attention-deficit hyperactivity disorder. Eur Child Adolesc Psychiatry 2015;24:105-113.

5) de Waal FB. Putting the altruism back into altruism: the evolution of empathy. Annu Rev Psychol 2008;59:279-300.

6) de Vignemont F, Singer T. The empathic brain: how, when and why? Trends Cogn Sci 2006;10:435-441.

7) Shamay-Tsoory SG. The neural bases for empathy. Neuroscientist 2011;17:18-24.

8) Decety J, Michalska KJ. Neurodevelopmental changes in the circuits underlying empathy and sympathy from childhood to adulthood. Dev Sci 2010;13:886-899.

9) Miller SR, Miller CJ, Bloom JS, Hynd GW, Craggs JG. Right hemisphere brain morphology, attention-deficit hyperactivity disorder (ADHD) subtype, and social comprehension. J Child Neurol 2006; 21:139-144

10) Kwak KJ, Oh SW, Kim CT. Korean-Wechsler Intelligence Scale for Children. 4th ed. Seoul: Inpsyt;2011. p.11-13.

11) Kim YS, Cheon KA, Kim BN, Chang SA, Yoo HJ, Kim JW, et al. The reliability and validity of Kiddie-Schedule for Affective Disorders and Schizophrenia-Present and Lifetime Version- Korean version (K-SADS-PL-K). Yonsei Med J 2004;45:81-89.

12) Pappas D. ADHD Rating Scale-IV: checklists, norms, and clinical interpretation. J Psychoeduc Assess 2006;24:172-178.

13) Davis MH. Measuring individual differences in empathy: evidence for a multidimensional approach. J Pers Soc Psychol 1983;44:113126.

14) Pfeifer JH, Iacoboni M, Mazziotta JC, Dapretto M. Mirroring others' emotions relates to empathy and interpersonal competence in children. Neuroimage 2008;39:2076-2085.

15) Ghim HR, Son JW, Eom JS, Jung MS, Park M, Park YO. Children's facial mimicry to dynamic emotional facial expressions and their emotional empathy. Korean J Cogn Biol Psychol 2012;24:433-452.

16) Fischl B, Dale AM. Measuring the thickness of the human cerebral cortex from magnetic resonance images. Proc Natl Acad Sci U S A 2000;97:11050-11055.

17) Groen Y, den Heijer AE, Fuermaier ABM, Althaus M, Tucha O. Reduced emotional empathy in adults with subclinical ADHD: evidence from the empathy and systemizing quotient. Atten Defic Hyperact Disord 2018;10:141-150.

18) Marton I, Wiener J, Rogers M, Moore C, Tannock R. Empathy and social perspective taking in children with attention-deficit/hyperactivity disorder. J Abnorm Child Psychol 2009;37:107-118.

19) Nilsen ES, Mewhort Buist TA, Gillis R, Fugelsang J. Communicative perspective-taking performance of adults with ADHD symptoms. J Atten Disord 2013;17:589-597.

20) Lockwood PL. The anatomy of empathy: vicarious experience and disorders of social cognition. Behav Brain Res 2016;311:255-266.

21) Goerlich-Dobre KS, Lamm C, Pripfl J, Habel U, Votinov M. The left amygdala: a shared substrate of alexithymia and empathy. Neuroimage 2015;122:20-32.

22) Kral TRA, Solis E, Mumford JA, Schuyler BS, Flook L, Rifken K, et al. Neural correlates of empathic accuracy in adolescence. Soc Cogn Affect Neurosci 2017;12:1701-1710.

23) Li Y, Zhang T, Li W, Zhang J, Jin Z, Li L. Linking brain structure and activation in anterior insula cortex to explain the trait empathy for pain. Hum Brain Mapp 2020;41:1030-1042.

24) Lamm C, Decety J, Singer T. Meta-analytic evidence for common and distinct neural networks associated with directly experienced pain and empathy for pain. Neuroimage 2011;54:2492-2502.

25) Mackes NK, Golm D, O'Daly OG, Sarkar S, Sonuga-Barke EJS, Fairchild G, et al. Tracking emotions in the brain-revisiting the empathic accuracy task. Neuroimage 2018;178:677-686.

26) Uribe C, Puig-Davi A, Abos A, Baggio HC, Junque C, Segura B. 
Neuroanatomical and functional correlates of cognitive and affective empathy in young healthy adults. Front Behav Neurosci 2019; 13:85.

27) Hoogman M, Muetzel R, Guimaraes JP, Shumskaya E, Mennes M, Zwiers MP, et al. Brain imaging of the cortex in ADHD: a coordinated analysis of large-scale clinical and population-based samples. Am J Psychiatry 2019;176:531-542.

28) Nakao T, Radua J, Rubia K, Mataix-Cols D. Gray matter volume abnormalities in ADHD: voxel-based meta-analysis exploring the effects of age and stimulant medication. Am J Psychiatry 2011; 168:1154-1163.

29) Volkow ND, Wang GJ, Tomasi D, Kollins SH, Wigal TL, Newcorn $\mathrm{JH}$, et al. Methylphenidate-elicited dopamine increases in ventral striatum are associated with long-term symptom improvement in adults with attention deficit hyperactivity disorder. J Neurosci 2012;32:841-849.

30) Villemonteix T, De Brito SA, Kavec M, Balériaux D, Metens T, Slama H, et al. Grey matter volumes in treatment naïve vs. chronically treated children with attention deficit/hyperactivity disorder: a combined approach. Eur Neuropsychopharmacol 2015;25:11181127.

31) Oakes HV, DeVee CE, Farmer B, Allen SA, Hall AN, Ensley T, et al. Neurogenesis within the hippocampus after chronic methylphenidate exposure. J Neural Transm (Vienna) 2019;126:201-209.

32) Plessen KJ, Bansal R, Zhu H, Whiteman R, Amat J, Quackenbush GA, et al. Hippocampus and amygdala morphology in attentiondeficit/hyperactivity disorder. Arch Gen Psychiatry 2006;63:795807. 